Derby his fine natural history collection, and the Museum of Archæology and Art, formed with so much care and cost, by Mr. Meyer, of Bebington.

Previous to Sir William Brown's princely gift little seems to have been done to advance education in Liverpool since 1647, when it was entered in the records, "Ordered that two dictionaries be provyed for the use of this towne and to be chayned." In I86I Sir William Brown stated that the Free Library and Museum would not be complete until it had attached to it a School of Science, which scheme was supported by the then Mayor, Mr. S. R. Graves, and the school was opened in October of that year by Lord Granville, then Lord President of the Council, supported by the Right Hon. W. E. Gladstone and Sir William Fairbairn, president of the British Association for that year. The school was affiliated with the Museum and Library Committee of the Corporation, and at present contains no less than $80 \mathrm{I}$ students, the largest number in any science school in the kingdom. Last year it obtained three scholarships at the Normal School of Science, South Kensington, and four Whitworth scholarships, whilst 8I per cent. of the students passed the Government Science Examinations, winning four Queen's medals and 233 Queen's prizes, and a Government Grant of 694l. In 1865 a period of depression occurred, which ceased in 1868, when Messrs. S. Leigh-Gregson and T. J. Moore, the indefatigable honorary secretaries, made vigorous efforts to improve the attendance, which was most successfully effected by doubling the fees, since when the marked prosperity has been unbroken, and all that is wanted is a central building instead of the present twelve centres of instruction.

A very valuable outcome of this School of Science are the free lectures, given at the expense of the Liverpool Corporation every winter in the Free Library and Museum, on subjects connected with the objects and specimens in the building. The programme for the present session includes numerous lectures on purely scientific subjects; amongst the lecturers are Messrs. Clements Markham, Lant Carpenter, De Rance, Professors R. S. Ball and Campbell Brown.

In addition to the Science School affiliated with the Corporation are the Liverpool Science and Art Classes, established in 1870 , through the energy of Mr. J. Samuelson. There are no less than fifty-one classes held in seven centres; the numbers of students in science are steadily increasing, and by arrangement with the Liverpool School Board their pupil-tearhers are allowed to attend the science classes, so as to qualify them for scienceteachers. In some cases laboratories have been erected at the Board schools, and the School Board have appointed a Science Demonstrator with two assistants of their own. The boys so taught have recently been examined by Prof. Forbes, of the Andersonian University, Glasgow, who reports very favourably of them.

Three years ago it was realised that the existing institutions did not satisfy the requirements of high education in Liverpool, and it was determined to found a University College, in connection with the Victoria University, and in this comparatively short time no less than seven Professors' Chairs have been endowed, with ten thousand pounds each, several chairs being founded by single donors, amongst whom is Lord Derby.

The Council of the College having no large funds to expend in the erection of imposing buildings, have been fortunate to receive from the City Council a large building standing in its own grounds at Brownlow Hill, a site on the brow of the hill 200 feet above the sea, overlooking the public buildings of Liverpool and the broad channel of the Mersey; in the city, yet removed from its traffic and turmoil. The building was originally erected at a cost of $20,000 \mathrm{l}$. ; it contains a rectangular centre with two wings; the solidity with which it was built has enabled Mr. Waterhouse to throw down the compartment walls of the wings, and to convert them into two lecture theatres, holding I 50 students each, while the body of the buildings form council, professors' and class rooms, whilst other rooms bave been fitted up as libraries, natural history, and physical laboratories, and engineering and drawing rooms. There are at present no chemical laboratories, but those of the adjacent medical school being available they are hardly required. The scientific chairs at present filled are those of Physics, Biology and Geology, and Chemistry, held by Professors Lodge, D.Sc., Herdman, D.Sc., F.L.S., and Campbell Brown, F.C.S. respectively. It is to be hoped ere long the wide range of knowledge covered by such sciences as biology, botany, and geology will be separated into at least two chairs, especially as the proximity of the Lancashire and Welsh coalfields render it important that Practical Geology and Mining should find a place amongst the College Chairs.

At the inaugural ceremony on Saturday Lord Derby presided, and showed from the sums that were still coming in that there is likely to be no lack of funds. Among other things he said: "We live in changing times, but democracy appreciates education, and especially appreciates science, and I think the scientific foundation is pretty safe not to be disendowed whatever happens. Let me add only this. Over and above its special educational work our college will do two things. It will be the centre of local patriotism, the means by which local public spirit may freely display itself, and it will give fresh evidence, if evidence is needed, that commerce and culture, so far from being antagonistic, are natural allies. They were so in Athens, they were so in Alexandria, they were so in the Italian Republics of the Middle Ages, and I do not think that a probably busier existence and a certainly smokier atmosphere constitute any reason why they should not be so here likewise."

Prof. Rendall, M.A., in delivering the inaugural address, said :- "The effort inaugurated that day was but one of many, each one wearing, indeed, its distinctive features, but all alike. What meant the simultaneous stir in Birmingham, in Bristol, in Leeds, in Nottingham, in Sheffield? What meant a host of cognate efforts in country towns and in the metropolis, too numerous to recapitulate?. What meant the sudden expansion in want of a college whose fate and trial it was to wait long and work obscurely, sustained rather by belief in its mission than by reassurances of success ? It was easy to say that the forwardness of founders was out of proportion to the zeal of students. As a matter of fact, founders' munificence has met with quick response, and five times out of six it is lack of funds, not lack of classes, that has hindered progress or even entailed defeat; and for predicting a like conclusion there were ample grounds. For primary education in England efficient provision had been made; of secondary and higher education the supply was sparse and capricious; while academic training remained the monopoly of the privileged and the wealthy. Unless the middle classes looked to it they would shortly find their children starting the race of life less well equipped for the inevitable struggle than those who in wealth and social standing have occupied a lower place."

Certainly the Liverpool College has made a most promising start; and considering the wealth of the city, there is no reason why, in a very short time, it should not be in perfect working order.

\section{BJERKNES' HYDRODYNAMIC EXPERIMENTS}

I N NATURE, vol. xxiv. p. 360 , were described in general terms the very interesting experimental researches of Prof. Bjerknes, of Christiania, which excited so much attention at the late Electrical Exhibition at Paris. Our readers will remember that the main point in those re- 
searches was the imitation of the phenomena of magnetic and elcctrical attraction and repulsion by analogous attractions and repulsions produced between pulsating or vibrating bodies immersed in liquid. The extreme im. portance of such experiments in hydrodynamical theory was so well pointed out by Prof. George Forbes in the former article, that nothing need be said here in that respect. The present article is confined to a concise deseription of the apparatus of M. Bjerkncs, and of the results obtained by its means.

Fig. I depicts the fundamental piece of apparatus for showing the action letween two pulsating drums or tambours, $A$ and 1 . These tambours consist of metal cups covered with an elastic membrane. Fach tambour communicates by a tube with an apparatus by moans of which

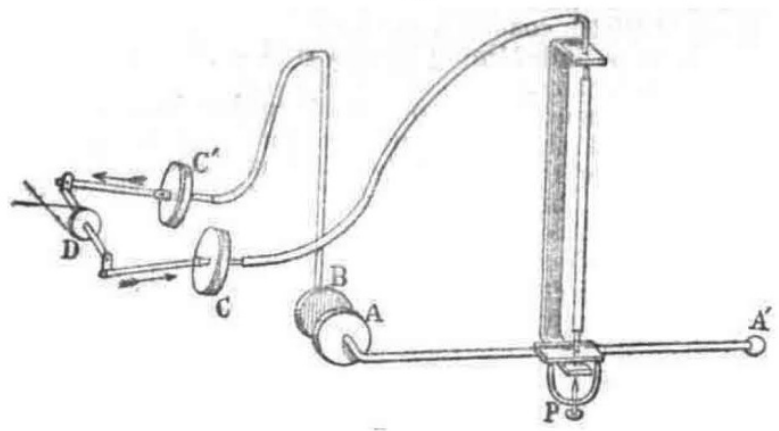

Fic. 1.

the elastic membrane is set in regular pulsation. A pulley, D, driven by a band from a multiplying wheel, works two small cranks whose rotations are converted into rectilinear movements by connecting-rods attachec! to two otber tambours, $C$ and $C^{\prime}$; the latter serving as pumps to alternately compress and rarefy the air in the tubes which communicate with $\mathrm{A}$ and B. Fig. 2 shows the construction of a single pulsating tambour. When air is compressed into it the membrane is forced outwards as at $\mathrm{C}$, when the pressure is withdrawn the membrane is drawn in as at D. Returning to the particular apparatus of Fig. I the result of setting the adjacent tambours in synchronous vibration when the whole apparatus is immersed in water, is as follows. If the tambours are arranged so that the movements are in similar phases

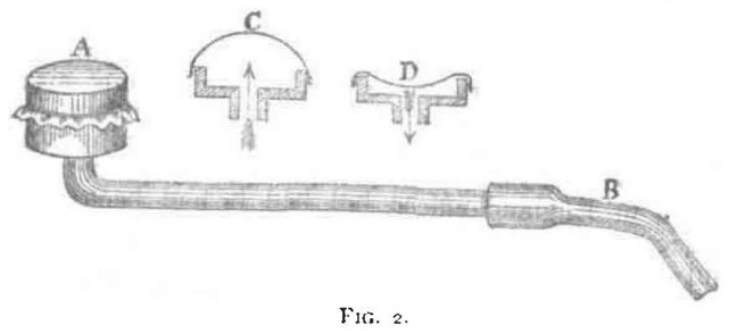

they attract one another; if in opposite phases they repel. This is exactly the inverse of what occurs for magnetic poles or electric charges, where similars repel one another and dissimilars attract one another. 'The tambour $\Lambda$ is mounted upon a lever capable of turning upon a pivot at $P$, and balanced by a counterpoise at $A^{\prime}$. The connection with the pump $\mathrm{C}$ is made through a vertical tube of india-rubber which permits of limited rotation about a vertical axis. The tambour $B$ is held in the hand at a convenient distance, just as one may hold in the hand a magnet to show its action upon a balanced compass needle.

A second fundamental piece of apparatus is the oscillating sphere shown in Fig. 3. To a pulsating tambour is fixed a small plate of metal, $c$, bearing a stiff wire terminated by a small ball, $\mathrm{A}$, and supported by a metal guide. The oscillations executed by such a ball when pulsations of air are directed into the tube $D$, are of course rectilinear displacements to and fro. Such an oscillating sphere presents at opposite sides opposite phases of displacement. Accordingly if the tube $I$, be connected with the pumping apparatus, and a tambour, such as that just described, communicating with the other pump, be held near the sphere $A$, it is found that the effects are of two opposite kinds, according to the position of the tambour. The oscillating sphere resembles then a magnet in having two poles of opposite propertics. These figures are from sketches furnished some months ago by M. Bjerknes. His apparatus, as shown in the Paris Exhibition, included a more powerful means of producing the pulsations. In Fig. 4 , which shows the tank and the collection of small pieces of apparatus, the driving gear of the pumps is not shown; but the pumps themselves are drawn in the lower right-hand corner of the figure, and consist of two small metal cylinders fitted with pistons; the connecting gear being so arranged that their movements can be made at will either in similar or in opposite directions. In the figure the fundamental experiment of the mutual action of two pulsating tambours is being shown. Two pulsating elastic spheres show similar results, but are less easy to manage. Some of the other portions of apparatus comprised in the collection are separately shown in Fig. 5. Of these the first is a double tambour whose two faces execute pulsations of similar phase. The second is a double tambour, the two chambers of which communicate separately with the two

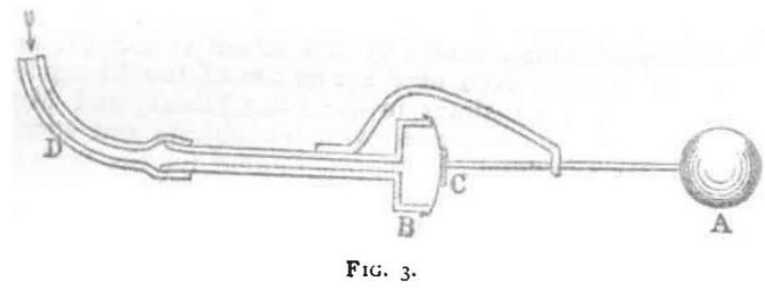

pumps, and in which therefore the two membranes execute movements in opposite phases. Thirdly, we have two spheres, of which one (on the left) is mounted so as to execute horizontal oscillations; the other (on the right) to oscillate vertically. It is possible to mount the oscillating spheres, either singly, or in pairs, upon horizontal axes, in which positions they act like mounted compass needles, following the action of another pulsating body; but always with the inversion of phase alluded to previously, like phase producing attraction; unlike, repulsion.

In the forcgoing cases the pulsating and oscillating bodies act on one another, producing (inversely) mutual actions and reactions as the poles of magnets do. $M$. Bjerknes has also succecded in imitating the phenomena of diamagnetism and of magnetic induction.

Diamagnetism is- imitated by making the pulsating bodies act upon objects lighter than water suspended within the liquid by a thread attached to a weight. A small ball of cork thus suspended is repelled from both a pulsator and an oscillator in whatever phase the latter may move. Temporary induced magnetism is imitated by the bchaviour of balls of some material heavier than water suspended from a float. Such a body is attracted by a vibrating or pulsating body. In the nearest corner of the tank of Fig. 4 are a pair of heavy bodies hung to threads for this very experiment. A little further to the right is shown a support from the top of which is suspended a little cylinder of heavy metal. When the pulsating tambour is held near this it turns round and points towards the tambour as a suspended piece of soft iron does towards a magnet pole. The same support carries a 
lower arm, from which a thread passes up to a cylinder bismuth or other diamagnetic body when placed between made lighter than water. This cylinder sets itself broad- the poles of an electromagnet. Here the importance of side to a pulsating tambour, behaving as a piece of the medium in determining by its own density the move-

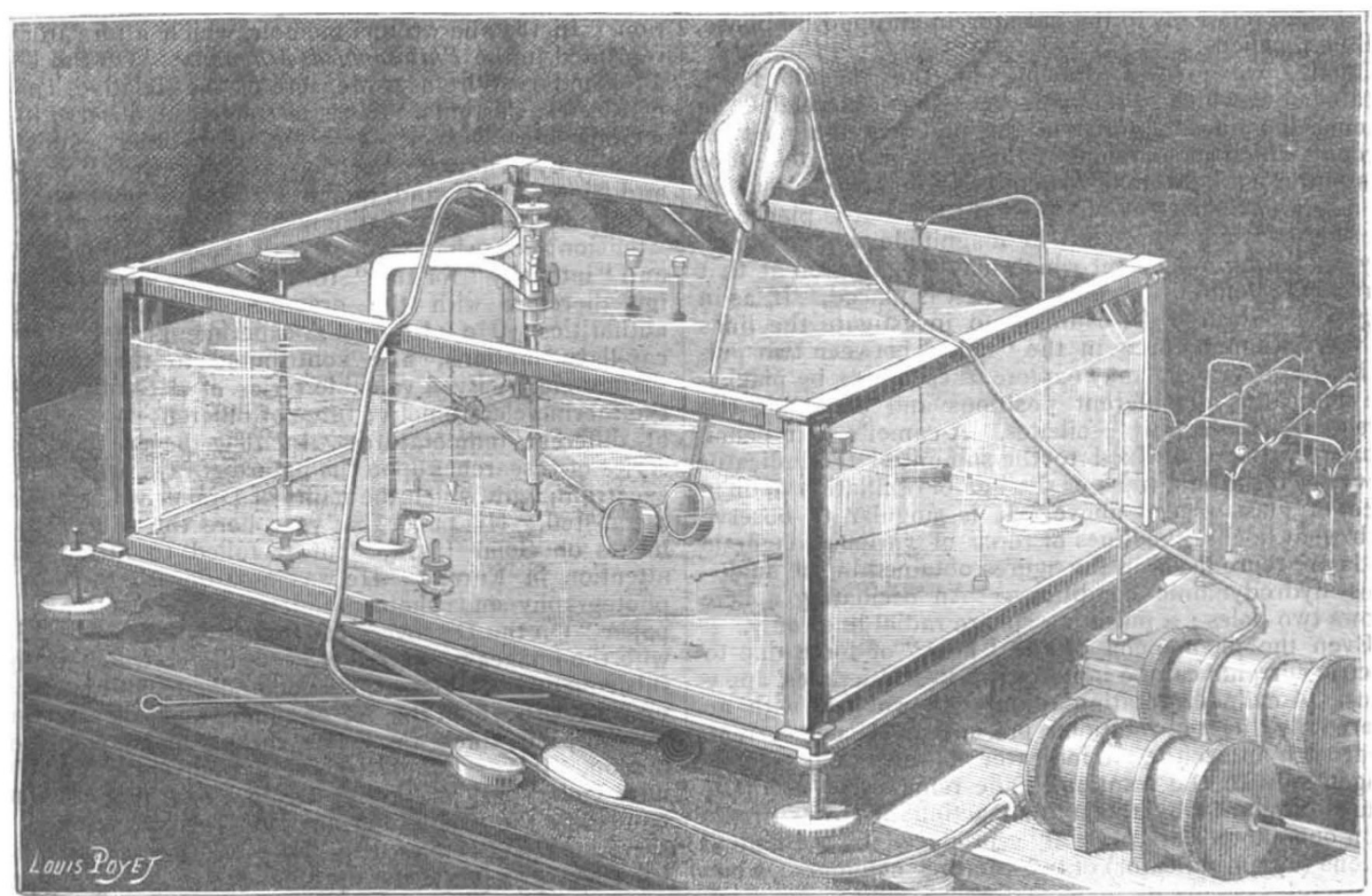

Fเc. 4 .

ments of the body acted upon, is obvious; and it furnishes an admirable commentary on the views of Faraday and Becquerel on the relation of the magnetic powers of the surrounding medium to the magnetic or diamagnetic propertics of bodics.

This particular series of experiments is most instructive;

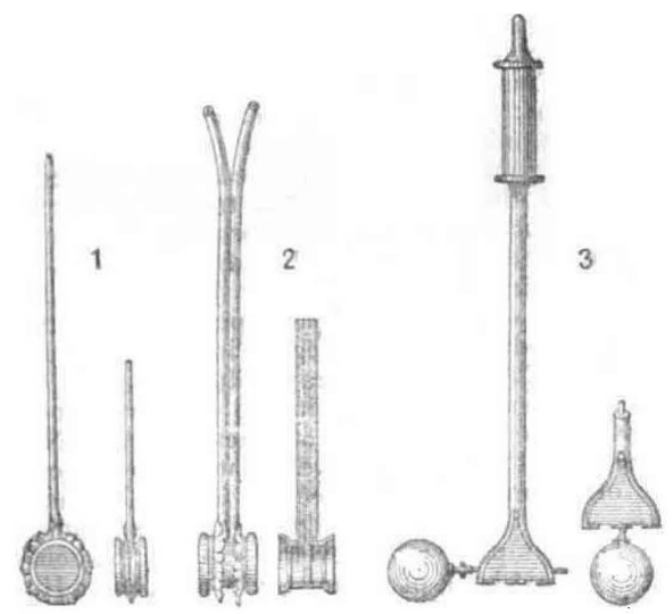

Fเ. 5.

and descrves a little further elucidation. If a small heavy ball hung to a float is acted upon by two pulsating tambours whose phases are opposite, it is a question in which phase the little ball shall vibrate. Eventually it takes up that of the more powerful pulsation, if they be of uncqual power, or of the nearcr one if they are unequally distant. The effect is like that on a small suspended bit of iron between two magnet poles of unequal strength, or at unequal distances. Now when a small hearzy body is set

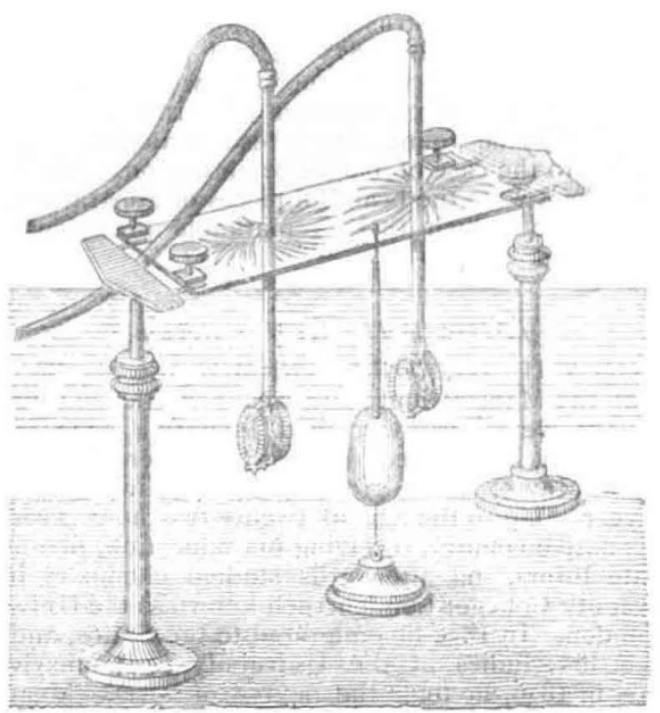

Fic. G.

pulsating by motions in the surrounding water it moves through a less amplitude than the water would have done in its place; for if it receive the same kinetic energy from 
the medium, its own mass being greater, its velocity will be less; its motions will therefore, relatively to the pulsator that is acting on it be in the same phase, and it will be attracted. On the other hand a body lighter than the surrounding water vibrates with a greater amplitude ; and being, relatively to the pulsator, in an opposite phase, will be repelled.

Lastly, we give a drawing of the ingenious indicator by which Prof. Bjerknes has succeeded in tracing the lines of force in the midst of the medium through which such actions as these are propagated. For this purpose a light hollow metal egg-shaped vessel held by a thin flexible steel wire to a heavy foot is placed in the tank. It takes up, both in magnitude and direction, any oscillating movement which may be going on at that part of the liquid in the tank where it is placed. If, as in Fig. 6, it is desired, for example, to investigate the lines of hydrodynamic force in the "field" between two pulsating tambours, we may explore these lines by placing the indicator at different positions and observing the direction in which it oscillates. A camel's hair pencil charged with colour fixed to the summit of the indicator serves to inscribe a trace of the line of oscillation upon a sheet of glass placed above. It is singular to observe with what fidelity the lines of force of various magnetic fields are reproduced in the figures obtained in the analogous hydrodynamic conditions. An oscillating sphere shows two poles; a pulsating sphere radial lines only.

Even the phenomena of the "field" of force due to electric currents can be imitated by Prof. Bjerknes' apparatus. A cylinder rotating round its own axis with an alternately directed rotation represents an electric current. Near such an excitor an indicator in the liquid vibrates tangentially to the rotation. The remarkable magnetic figures produced by the mutual action of two currents upon one another are perfectly reproduced by the mutual actions (on an indicator) of two rotating cylinders; while the spiral systems of lines of force produced, as Prof. Silvanus Thompson discovered (see NATURE, vol. xix. p. 83), by the mutual action of a magnet pole and of a current traversing it are exactly reproduced by Prof. Bjerknes' indicator under the influence of an apparatus which pulsates and rotates synchronously. The most extraordinary thing about Prof. Bjerknes' researches is that they are all the result not of haphazard experiment, but of careful and abstruse calculation. In 1865 he began the investigation. By 1875 he had perceived that the calculated motions were such as would have direct analogies with the phenomena of permanent magnets. Toward I 879 he found that these analogies might be extended to the case of magnetic induction. Then, and not till then, were the beautiful pieces of apparatus made, by means of which these remarkable previsions have been verified.

\section{JOHN WILLIAM DRAPER}

$\mathrm{J}^{\mathrm{o}}$ HN WILLIAM DRAPER, M.D., LL.D., President of the Medical and Scientific Faculties of the University of New York, who died January 4, I882, was an Englishman, having been born at St. Helens, near Liverpool, on May 5, I8II. He was therefore in his seventyfirst year. Up to the age of twenty-two he was resident in his native country, receiving his education, first under private tutors, and afterwards studied chemistry in the University College, London, then known as the University of London. In 1832 he emigrated to the States, and con. tinued his studies at the University of Pennsylvania, where, in 1836 , he took the degree of M.D. Meantime his talent for original research had manifested itself in the production of several memoirs, which appeared in the pages of the Journal of the Franklin Institution. The first of these (published in 1834) was "On the Nature of Capillary Attraction"; whilst a second was devoted to a discussion of the most eligible method of constructing galvanic batteries of four elements. In 1835 he published an account of some experiments made to detect whether light exhibits any. magnetic actions. Several branches of the science of electricity subsequently claimed his attention. In 1839 he wrote a memoir, which afterwards was reprinted in the Philosophical Magazine, "On the Use of a Secondary Wire as a Measure of the Relative Tension of Electric Currents." It is instructive to observe in this memoir how Draper's exact mind revolted against the misuse, by writers on electricity, of the words "tension" and "intensity"; and, though he himself employed both terms, he carefully distinguished between them, using "tension" for what we now call "electromotive force," and "intensity" for the "strength of the current," agreeing therefore with the practice of many continental authorities. He also made experiments upon electrocapillary motions, and contributed to the science of thermo-electricity, a valuable series of determinations of the thermo-electromotive force of different pairs of metals at different temperatures. In 1837 began the notable series of researches upon the nature of rays of light in the spectrum, with which the name of Draper will always be associated. His paper that year bore the title "Experiments on Solar Light," but it failed to attract much attention in Europe. He was now devoting himself to photography and photo-chemistry with great zeal. His paper "On the Discovery of Latent Light," in 1 842 , dealt with the images produced by rays of light which are only subsequently developed by some chemical reaction-a process with which the art of photography has made us familiar, but which was then a curious and novel phenomenon. It was Draper who first discovered that in the ultra-violet part of the spectrum there are absorption bands like the Fraunhofer lines in the visible part of the spectrum. To enumerate the works which proceeded from Draper's pen upon the chemical and physical properties of the ultra-violet, or as he styled them, tithonic rays, would be inadmissible here. Suffice it to say that the greater part of the fifty memoirs mentioned in the Royal Society's Catalogue related to this subject, and the most important of them are to be found reprinted in his "Scientific Memoirs," published in 1878 . In this volume may be found the pregnant suggestion for a standard of white light for photometry of a piece of platinum foil of given size and thickness, raised to a white heat by an electric current of specified strength. To guard against fusion he suggested that an automatic short-circuiting apparatus should be constructed by some "skilled artificer." He thus exactly anticipated Edison's first incandescent lamps: though the satisfactory standard of white light appears to be as far off as ever.

The latest papers Draper published were entitled "Researches in Actino-chemistry," and treated of the distribution of heat and of chemical force in the spectrum. They appeared in 1872 in the American Journal of Science and in the Philosophical Magazine. During these years of work Draper held important appointments, first in Hampden-Sidney College, Virginia, where he was Professor of Chemistry, Natural Philosophy, and Physiology, and afterwards (1839) in the University of New York, where he was Professor of Chemistry and Natural History, a post modified two years later into that of Professor of Chemistry in the Medical College of the University. In addition to the original memoirs enumerated above Dr. Draper wrote several valued text-books of science; a Text-book of Chemistry in 1846 , and a Human Physiology in 1856 , both of which works went through several editions.

Dr. Draper's literary activity manifested itself however in other directions, and he has left an enduring mark in literature as a philosophic historian of no mean merit. The "History of the Intellectual Development of Europe," published in 1862 , has been translated into all the current 